\title{
Mass sum rule of hadrons in the QCD instanton vacuum
}

\author{
Ismail Zahed ${ }^{*}$ \\ Center for Nuclear Theory, Department of Physics and Astronomy, Stony Brook University, \\ Stony Brook, New York 11794-3800, USA
}

(Received 8 March 2021; accepted 3 September 2021; published 24 September 2021)

\begin{abstract}
We briefly review the key aspect of the QCD instanton vacuum in relation to the quantum breaking of conformal symmetry and the trace anomaly. We use Ji's invariant mass decomposition of the energy momentum tensor together with the trace anomaly to discuss the mass budget of the nucleon and pion in the QCD instanton vacuum. A measure of the gluon condensate in the nucleon is a measure of the compressibility of the QCD instanton vacuum as a dilute topological liquid.
\end{abstract}

DOI: 10.1103/PhysRevD.104.054031

\section{INTRODUCTION}

A remarkable feature of QCD is that in the chiral limit, it is a scale free theory. Yet, all hadrons are massive, composing most of the visible mass in the universe. The typical hadronic scale is $1 \mathrm{fm}$, but where does it come from? The answer appears to be from a subtle quantum effect referred to as dimensional transmutation and related to the quantum breaking of the conformal symmetry of QCD. This mechanism is nonperturbative. On the lattice, the lattice cutoff along with the running coupling combine to generate this scale. In the continuum, to achieve this mechanism requires a nonperturbative description of the vacuum state and its excitations.

The QCD vacuum as a topological liquid of instantons and anti-instantons, offers by far the most compelling nonperturbative description that is analytically tractable in the continuum, thanks to its QCD semiclassical origin and diluteness [1-3]. It is not the only description. Other candidates based on center vortices and monopoles, to cite a few [4], are also suggested and may as well be present in addition to the instantons. However, the latter appears to trigger the dual breaking of conformal and chiral symmetry breaking and dominate the vacuum state and its low-lying hadronic excitations. Center vortices may be important for the disordering of the large Wilson loops and confinement, a mechanism likely at work in the orbitally excited hadrons as they Reggeize.

The spontaneous breaking of chiral symmetry rather than confinement drives the formation of the low-lying and

\footnotetext{
*ismail.zahed@stonybrook.edu
}

Published by the American Physical Society under the terms of the Creative Commons Attribution 4.0 International license. Further distribution of this work must maintain attribution to the author(s) and the published article's title, journal citation, and DOI. Funded by SCOAP ${ }^{3}$. stable hadrons such as the nucleon and pion. In the QCD instanton vacuum, conformal symmetry is broken by the density of instantons: their continuous quantum rate of tunneling in the vacuum. The breaking of chiral symmetry follows from the delocalization of the light quark zero modes by leapfrogging the instantons and anti-instantons much like electrons leapfrog atoms in a metal. Detailed numerical simulations of light hadronic correlators in the QCD instanton vacuum [5] show remarkable agreement with direct lattice measurements [6] and a wealth of correlators extracted from data [5]. The universal conductance fluctuations in the zero mode region of the Dirac spectrum, predicted by random matrix theory [7] and confirmed by lattice simulations [8], show unequivocally the topological character of the origin of mass.

In this note, we briefly review the salient aspects of the QCD instanton vacuum in relation to the quantum breaking of conformal symmetry in Sec. II. We then discuss the role of the trace anomaly in the nucleon and pion mass in Sec. III. The quark and gluon composition of the hadronic mass using Ji's decomposition [9] is discussed in Sec. IV. In Sec. V, we show that the gluon condensate in the nucleon is tied to the QCD vacuum compressibility, a measure of the diluteness of the QCD instanton vacuum as a topological liquid. Our conclusions are in Sec. VI.

\section{QCD INSTANTON VACUUM}

As we noted above, the chief aspect of the $\mathrm{QCD}$ vacuum (meaning quenched throughout) is its quantum breaking of conformal symmetry, with the emergence of all light hadronic scales. The nature of the gauge fields at the origin of this breaking were mysterious and the subject of considerable debates and speculations for many decades until stunning pictures were developed by Leinweber and his collaborators using cooling and/or projection techniques $[10,11]$. Out of the fog of millions of gauge 


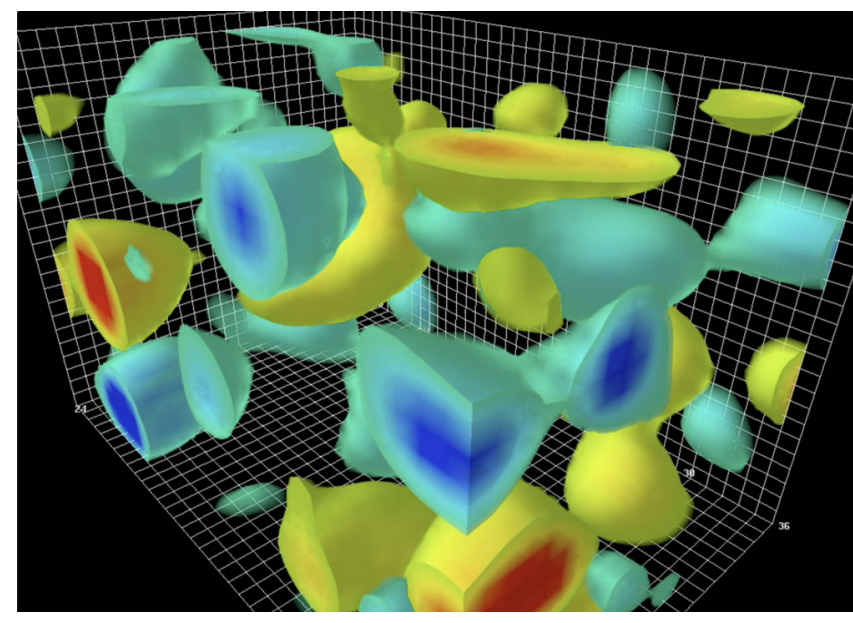

FIG. 1. Instantons (yellow) and anti-instantons (blue) configurations in the cooled Yang-Mills vacuum [10]. They constitute the primordial gluon epoxy at the origin of the hadronic mass. See text.

fluctuations, cooling has revealed a stunning vacuum landscape composed of inhomogeneous and topologically active gauge fields as shown in Fig. 1. Remarkably, the key features of this vacuum were predicted long ago by Shuryak [12],

$$
n_{I+\bar{I}} \equiv \frac{1}{R^{4}} \approx \frac{1}{\mathrm{fm}^{4}} \quad \frac{\bar{\rho}}{R} \approx \frac{1}{3},
$$

for the instanton plus anti-instanton density and size, respectively. In other words, the hadronic scale $R=$ $1 \mathrm{fm}$ emerges as the mean quantum tunneling rate of the topological charge in the QCD vacuum. The dimensionfull parameters (1) combine in the dimensionless packing parameter $\kappa \equiv \pi^{2} \bar{\rho}^{4} n_{I+\bar{I}} \approx 0.1$, a measure of the diluteness of the instanton-anti-instanton ensemble in the QCD vacuum. Fortunately, the smallness of $\kappa$ is what will allow us to do reliable analytical calculations. In the cooled landscape shown in Fig. 1, most hadronic correlations are left unchanged with those before cooling [2] (and references therein). The size distribution of the instantons and anti-instantons density (their tunneling rate per size) in the QCD vacuum is well captured semiempirically by

$$
\frac{d n(\rho)}{d \rho} \sim \frac{1}{\rho^{5}}\left(\rho \Lambda_{\mathrm{QCD}}\right)^{b} e^{-\# \rho^{2} / R^{2}},
$$

with $b=11 N_{c} / 3-2 N_{f} / 3$ (one loop).

The quantum breaking of conformal symmetry is best captured through the trace of the energy momentum tensor. Indeed, consider its symmetric form,

$$
\begin{aligned}
T^{\mu \nu} & =\frac{2}{\sqrt{-g}} \frac{\delta S_{1+3}}{\delta g_{\mu \nu}} \\
& \left.=F^{a \mu \lambda} F_{\lambda}^{a \nu}-\frac{1}{4} g^{\mu \nu} F^{2}+\frac{1}{4} \bar{\psi} \gamma^{[\mu} i \stackrel{\leftrightarrow}{D}\right]_{+} \psi,
\end{aligned}
$$

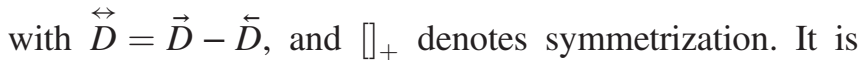
conserved $\partial_{\mu} T^{\mu \nu}=0$, with an anomalous trace,

$$
T_{\mu}^{\mu}=\frac{\beta\left(g^{2}\right)}{4 g^{4}} F_{\mu \nu}^{a} F^{a \mu \nu}+m \bar{\psi} \psi,
$$

with the Gell-Mann-Low beta-function (two loops),

$$
\beta\left(g^{2}\right)=-\frac{b g^{4}}{8 \pi^{2}}-\frac{\bar{b} g^{6}}{2\left(8 \pi^{2}\right)^{2}}+\mathcal{O}\left(g^{8}\right) .
$$

Throughout, we use the rescaling $g F \rightarrow F$ for all operators in the instanton and anti-instanton gauge fields. In the QCD instanton vacuum, the gluon operator $F^{2} /\left(32 \pi^{2}\right) \rightarrow$ $\left(N_{+}+N_{-}\right) / V$ counts the number of instantons plus antiinstantons per 4-volume $V$. In the canonical ensemble with zero theta-angle, it is fixed by the instanton density with $N_{ \pm} / V=\bar{N} / 2 V$. Therefore, we have

$$
\begin{aligned}
\left\langle T_{\mu}^{\mu}\right\rangle & \approx-b\left(\frac{\bar{N}}{V}\right)+m\langle\bar{\psi} \psi\rangle \approx-b\left(\frac{\bar{N}}{V}\right)(1+\mathcal{O}(m R)) \\
& \approx-10 \mathrm{fm}^{-4}
\end{aligned}
$$

setting the scale of all hadrons. The current mass $m \approx$ $8 \mathrm{MeV}$ in (6) is fixed at the soft renormalization point $\bar{\rho} \approx 0.3 \mathrm{fm}$, about twice the commonly used value at the hard renormalization scale. The scale of the spontaneous breaking of chiral symmetry is also fixed by the finite instanton density, but its contribution to the vacuum scalar density is small since $m R \approx(8 \mathrm{MeV})(1 \mathrm{fm}) \approx 1 / 25$.

Some of the quantum scale fluctuations in QCD are captured in the QCD instanton vacuum using the grandcanonical description instead of the canonical one. In the former, the instanton number $N=N_{+}+N_{-}$is allowed to fluctuate with the measure $[3,13,14]$,

$$
\mathbb{P}(N)=e^{\frac{b N}{4}}\left(\frac{\bar{N}}{N}\right)^{\frac{b N}{4}}
$$

which is stronger than Poisson $(b / 4 \rightarrow 1)$, to reproduce the vacuum compressibility,

$$
\frac{\left\langle(N-\bar{N})^{2}\right\rangle_{\mathbb{P}}}{\bar{N}}=\frac{4}{b},
$$

expected from QCD low-energy theorems [15].

\section{MASS IDENTITY}

We now focus on the anomalous trace of the QCD energy momentum tensor and its relation to the hadron mass. It is worth stressing that the ensuing relation to the mass is just a bulk identity and not a mass decomposition. Having said that, the trace couples to a scalar dilaton, which sources a sigma (two-pion) meson and/or a $0^{++}$scalar 
glueball field. QCD perturbative arguments suggest that this trace may be accessible in the photo-production of charmonium at treshold [16,17] (and references therein), although the coupling to the $2^{++}$tensor glueball may still be very active in the threshold region [18]. Recall that the Reggeized form of the $2^{++}$exchange transmutes to the Pomeron and is dominant asymptotically.

\section{A. Nucleon}

For a nucleon state $|P\rangle$ with the standard normalization $\left\langle P \mid P^{\prime}\right\rangle=2 E_{P}(2 \pi)^{3} \delta^{3}\left(P-P^{\prime}\right)$, one has

$$
\left\langle P\left|T^{\mu \nu}\right| P\right\rangle=2 P^{\mu} P^{\nu},
$$

with the trace in any frame (one loop),

$$
\left\langle P\left|T_{\mu}^{\mu}\right| P\right\rangle=\left\langle P\left|\left(-\frac{b}{32 \pi^{2}} F^{2}+m \bar{\psi} \psi\right)\right| P\right\rangle=2 M_{N}^{2},
$$

with $g^{2} F^{2} \rightarrow F^{2}$ for the strong instanton and anti-instanton gauge fields. It is renormalization group invariant. The identity (10) shows that the nucleon mass is the change of the conformal anomaly or gluon field in a nucleon state. However, the formation of the state occurs only if chiral symmetry is spontaneously broken as we noted earlier. (10) is a QCD identity that is satisfied in the QCD instanton vacuum as we now show.

In the rest frame, the gluon contribution in (10) follows from the normalized and connected three-point function asymptotically,

$$
\frac{\left\langle P\left|F^{2}\right| P\right\rangle}{\langle P \mid P\rangle}=\lim _{T \rightarrow \infty} \frac{\left\langle J_{P}^{\dagger}(T) F^{2} J_{P}(-T)\right\rangle_{C}}{\left\langle J_{P}^{\dagger}(T) J_{P}(-T)\right\rangle}
$$

with $J_{P}$ a pertrinent nucleon source. In the canonical description of the QCD instanton vacuum, $F^{2} /\left(32 \pi^{2}\right) \rightarrow$ $\bar{N} / V$ is a number. It factors out in the three-point correlator in (11) (numerator), and the connected correlator vanishes.

A nonvanishing contribution to the connected threepoint correlator follows from the grand-canonical description, where $N$ is allowed to fluctuate as we noted in (7). With this in mind, it is straightforward to see that (11) is dominated by the variance,

$\frac{V}{32 \pi^{2}} \frac{\left\langle P\left|F^{2}\right| P\right\rangle}{\langle P \mid P\rangle} \approx\left\langle(N-\bar{N})^{2}\right\rangle_{\mathbb{P}} \frac{\partial}{\partial \bar{N}} \log \left(\lim _{T \rightarrow \infty}\left\langle J_{P}^{\dagger}(T) J_{P}(-T)\right\rangle\right)$,

with the higher moments suppressed by $1 / b^{2} \sim 1 / N_{c}^{2}$. The result (12) was noted in [13] (see Eq. 5.8) using a fermionization method and in [14] [see Eqs. (91) and (93)] using a bosonization method, each of the QCD instanton vacuum in the $1 / N_{c}$ approximation. The expectation value in the first bracket is carried using the distribution (7). (12) illustrates how the nucleon scoops the epoxy from the QCD instanton vacuum.

All dimensions in the QCD instanton vacuum are fixed by the density $\bar{N} / V=1 / R^{4}$ and the current quark masses. The nucleon mass is the sum of the chirally symmetric (invariant mass) plus the symmetry breaking contribution (pion-nucleon sigma term),

$M_{N}=M_{\mathrm{inv}}+\sigma_{\pi N}=C\left(\frac{\bar{N}}{V}\right)^{\frac{1}{4}}+\bar{C} m(1+\mathcal{O}(m R))$,

with [19-21]

$$
\sigma_{\pi N}=\frac{\langle P|m \bar{\psi} \psi| P\rangle}{\langle P \mid P\rangle} \approx 50 \mathrm{MeV}
$$

evaluated at the soft renormalization scale $\bar{\rho}=0.6 \mathrm{GeV}$, which is the appropriate scale for hadronic spectroscopy. The right-most relation in (13) follows from the QCD instanton vacuum. As a result, the anomalous contribution in the QCD instanton vacuum is

$$
\frac{V}{2 T} \frac{-b}{32 \pi^{2}} \frac{\left\langle P\left|F^{2}\right| P\right\rangle}{\langle P \mid P\rangle}=4 \frac{\partial M_{N}}{\partial \log \bar{N}}=M_{\mathrm{inv}},
$$

which is seen to satisfy the sum rule,

$$
\frac{\left\langle P\left|T_{\mu}^{\mu}\right| P\right\rangle}{2 M_{N}}=M_{\mathrm{inv}}+\frac{\langle P|m \bar{\psi} \psi| P\rangle}{2 M_{N}}=M_{N} .
$$

\section{B. Pion}

The preceding arguments apply also to the pion, with one major difference,

$$
m_{\pi}=C \sqrt{m}\left(\frac{\bar{N}}{V}\right)^{\frac{1}{8}}(1+\mathcal{O}(m R)),
$$

since it is a Goldstone mode. The $\mathcal{O}(m R)$ corrections are small in the QCD instanton vacuum. It follows that

$$
\frac{V}{2 T} \frac{-b}{32 \pi^{2}} \frac{\left\langle\pi\left|F^{2}\right| \pi\right\rangle}{\langle\pi \mid \pi\rangle}=4 \frac{\partial m_{\pi}}{\partial \log \bar{N}}=\frac{1}{2} m_{\pi},
$$

which was first observed in [22], with the sum rule,

$$
\frac{\left\langle\pi\left|T_{\mu}^{\mu}\right| \pi\right\rangle}{2 m_{\pi}}=\frac{1}{2} m_{\pi}+\frac{\langle\pi|m \bar{\psi} \psi| \pi\rangle}{2 m_{\pi}}=m_{\pi},
$$

satisfied, as expected. The pion sigma-term follows from chiral reduction or the Feynman-Hellmann theorem,

$$
\frac{\langle\pi|m \bar{\psi} \psi| \pi\rangle}{2 m_{\pi}}=\frac{\partial \mathcal{E}_{\pi}}{\partial \log m}=\frac{1}{2} m_{\pi} .
$$




\section{JI MASS SUM RULE}

The trace identity (9) reflects on the general fact that all hadron masses in QCD are tied to the quantum breaking of conformal symmetry as we noted earlier and should be enforced by any nonperturbative quantum description, whether numerical such as the lattice or analytical such as the QCD instanton vacuum. However, it does not specifically budget this mass breaking in terms of the hadron constituents. In a strongly interacting theory, this issue may be elusive, especially with a soft renormalization scale, as the gluons are strongly intertwined with the light quarks. This is more so in the unquenched and screened formulation.

This notwithstanding, a specific and physically motivated proposal to budget the mass was put forth by $\mathrm{Ji}$ in $[9,22]$ and since revisited by many [23-25] (and references therein). The ensuing mass composition involves the sum of partonic contributions, some of which may be measurable using DIS experiments. The proposal relies on an invariant decomposition of the energy momentum tensor, which we now detail.

The energy-momentum tensor (3) can be decomposed as the sum of a traceless and tracefull part [22,26],

$$
T^{\mu \nu} \equiv \bar{T}^{\mu \nu}+\hat{T}^{\mu \nu} \equiv \bar{T}^{\mu \nu}+g^{\mu \nu} \frac{1}{4} T_{\alpha}^{\alpha}
$$

where the traceless part reads

$$
\begin{aligned}
\bar{T}^{\mu \nu}= & \left(-F^{a \mu \tau} F_{\tau}^{a \nu}+\frac{1}{4} g^{\mu \nu} F^{2}\right) \\
& +\frac{1}{4} \bar{\psi} \gamma^{[\mu} i \stackrel{\leftrightarrow}{\left.D^{\nu}\right]_{+}} \psi-g^{\mu \nu} \frac{1}{4} m \bar{\psi} \psi,
\end{aligned}
$$

and the tracefull part is

$\hat{T}^{\mu \nu}=g^{\mu \nu} \frac{1}{4}\left(\frac{\beta\left(g^{2}\right)}{4 g^{4}} F^{2}+m \bar{\psi} \psi \approx-\frac{b}{32 \pi^{2}} F^{2}+m \bar{\psi} \psi\right)$.

We note that this decomposition is commensurate with the analysis of the nucleon energy momentum tensor in holographic QCD through dual gravitons in bulk [18]. (Holography is a good example of a strong coupling description of a gauge theory via its gravity dual, where the partonic structure is elusive.)

The tracefull and traceless part of the energy momentum tensor (21)-(23) correspond to the spin-2 and spin-0 representations of the Lorentz group and do not mix under renormalization by symmetry. Their renormalization at the instanton size scale $\bar{\rho} \approx 0.3 \mathrm{fm}$ is subsumed throughout. On the lattice, this soft renormalization scale is best achieved using a cooling procedure, where only the UV quantum and nonsingular fluctuations are subtracted (our instantons are classical fields in singular gauge). Note that our renormalization scale is softer than the one used in currently fine lattices with $1 / \mu \approx 0.1 \mathrm{fm}$ ( $\overline{\mathrm{MS}}$ scheme) [27]. This difference will be further discussed below.

With this in mind, the matrix elements of the split energy-momentum tensor are constrained by Lorentz symmetry,

$$
\begin{aligned}
& \left\langle P\left|\bar{T}^{\mu \nu}\right| P\right\rangle=2\left(P^{\mu} P^{\nu}-\frac{1}{4} g^{\mu \nu} M_{N}^{2}\right) \\
& \left\langle P\left|\hat{T}^{\mu \nu}\right| P\right\rangle=\frac{1}{2} g^{\mu \nu} M_{N}^{2} .
\end{aligned}
$$

The corresponding Hamiltonian in Minkowski signature follows from the 00-component of (21)-(23) modulo BRST exact and gauge dependent contributions,

$$
\begin{aligned}
H_{G} & =\int d^{3} x \bar{T}_{G}^{00}=\int d^{3} x \frac{1}{2}\left(E^{2}+B^{2}\right) \\
H_{Q}^{\prime} & =\int d^{3} x \bar{T}_{Q}^{00}=\int d^{3} x\left(\frac{1}{2} \bar{\psi} \gamma \cdot i \stackrel{\leftrightarrow}{D} \psi+\frac{3}{4} m \bar{\psi} \psi\right) \\
H_{A}^{\prime} & =\int d^{3} x \hat{T}_{A}^{00} \\
& =\int d^{3} x \frac{1}{4}\left(\frac{\beta\left(g^{2}\right)}{4 g^{4}} F^{2}+m \bar{\psi} \psi \approx-\frac{b}{32 \pi^{2}} F^{2}+m \bar{\psi} \psi\right),
\end{aligned}
$$

where the time $t=0$ is subsumed. The mass term can be rearranged so that (25) reads

$$
\begin{aligned}
& H_{G}=\int d^{3} x \bar{T}_{G}^{00}=\int d^{3} x \frac{1}{2}\left(E^{2}+B^{2}\right) \\
& H_{Q}=\int d^{3} x \bar{T}_{Q}^{00}=\int d^{3} x\left(\frac{1}{2} \bar{\psi} \gamma \cdot i \stackrel{\leftrightarrow}{D} \psi\right) \\
& H_{A}=\int d^{3} x \hat{T}_{A}^{00}=\int d^{3} x \frac{1}{4}\left(\frac{\beta\left(g^{2}\right)}{4 g^{4}} F^{2} \approx-\frac{b}{32 \pi^{2}} F^{2}\right) \\
& H_{m}=\int d^{3} x \bar{T}_{G}^{00}=\int d^{3} x m \bar{\psi} \psi .
\end{aligned}
$$

The nucleon mass budget is then

$$
\begin{aligned}
M_{N} & =\frac{\left\langle P\left|H_{G}+H_{Q}+H_{A}+H_{m}\right| P\right\rangle}{\langle P \mid P\rangle} \\
& \equiv M_{G}^{N}+M_{Q}^{N}+M_{A}^{N}+M_{m}^{N},
\end{aligned}
$$

which shows that the combination,

$$
M_{\mathrm{inv}}=M_{G}^{N}+M_{Q}^{N}+M_{A}^{N},
$$

is chirally symmetric and equal to the invariant mass in (13).

In Euclidean signature, whether on the lattice or using the QCD instanton vacuum, (27) can be evaluated by 
trading $T^{00} \rightarrow T^{44}$ and $t=0 \rightarrow i 0$. In the dilute QCD instanton vacuum, the gluonic operator in (25)-(27) is the sum of multi-instanton contributions of the form,

$$
\begin{aligned}
\bar{T}_{G}^{44}[A] & =\sum_{I=1}^{N_{ \pm}} \bar{T}_{G}^{44}\left[A_{I}\left(\xi_{I}\right)\right]+\sum_{I \neq J=1}^{N_{ \pm}} \bar{T}_{G}^{44}\left[A_{I}\left(\xi_{I}\right), A_{J}\left(\xi_{J}\right)\right]+\cdots \\
& =\sum_{I \neq J=1}^{N_{ \pm}} \bar{T}_{G}^{44}\left[A_{I}\left(\xi_{I}\right), A_{J}\left(\xi_{J}\right)\right]+\cdots
\end{aligned}
$$

Since the first one-instanton contribution in (29) is composed of self-dual fields, it vanishes. So, we are left with only the two and higher multi-instanton contributions. When averaged over a measure of independent instantons, the remaining terms in (29) are suppressed by the diluteness factor $\kappa \approx 0.1$. As a result, the contribution of $M_{G}^{N}$ is parametrically small in comparison to $M_{Q}^{N}$ or $4 M_{A}^{N}$; i.e., $M_{G}^{N} / M_{Q}^{N} \approx \kappa \approx 0.1$. The contributions $M_{Q, m}^{N}$ are solely given in terms of the fermionic zero modes (modulo the instanton gauge fields in the long derivative).

With this in mind, the breakdown in the mass budget (27) for the nucleon yields the estimates,

$$
\begin{aligned}
& \frac{M_{Q}^{N}}{M_{N}} \approx \frac{3}{4} \frac{1}{1+\kappa}\left(1-\frac{\sigma_{\pi N}}{M_{N}}\right) \approx 64 \% \\
& \frac{M_{G}^{N}}{M_{N}} \approx \frac{3}{4} \frac{\kappa}{1+\kappa}\left(1-\frac{\sigma_{\pi N}}{M_{N}}\right) \approx 7 \% \\
& \frac{M_{A}^{N}}{M_{N}}=\frac{1}{4}\left(1-\frac{\sigma_{\pi N}}{M_{N}}\right) \approx 24 \% \\
& \frac{M_{m}^{N}}{M_{M}}=\frac{\sigma_{\pi N}}{M_{N}} \approx 5 \%,
\end{aligned}
$$

with the empirical pion-nucleon sigma term (14). (30) shows that in the QCD instanton vacuum, about $70 \%$ of the nucleon mass stems from the valence quarks (hopping zero modes), $25 \%$ from the gluon condensate or epoxy (displaced vacuum instanton field), and $7 \%$ from emerging valence gluons. The nucleon is composed mostly of quark constituents hopping and dragging the gluon epoxy. The gluon epoxy in the nucleon is the quantum anomalous energy in the nucleon discussed recently in [28].

We note that the budgeting of the nucleon mass in (30) differs from the one reported on the lattice in [27], with a noticeably larger valence gluon fraction in the lattice nucleon. In our analysis, this can only be accommodated by a stronger instanton packing fraction of $\kappa \approx 0.5$ instead of 0.1 . We note that larger values of $\kappa$ of about 1 , which account for close instanton-anti-instanton pairs not responsible for the breaking of chiral symmetry, are present at intermediate cooling and contribute to certain gluonic correlations all the way to zero cooling time [29]. The harder renormalization scale $\mu=2 \mathrm{GeV}$ used in the reported lattice results is the likely source of the valence and perturbative gluon enhancement reported in the lattice nucleon.

Quantum evolution will enhance $M_{G}^{N}$ at the expense of $M_{Q}^{N}$, which, in (30), would amount to effectively dressing $\kappa \approx 0.1 \rightarrow 0.5$ at $\mu=2 \mathrm{GeV}$. Indeed, Fig. 1 refers to a deeply cooled lattice configuration where smaller instanton-anti-instanton molecules (streamlines) are annihilated. When cooling is performed by the gradient flow method [30], the scale of these additional semiclassical contributions can be made more precise using the renormalization group flow. Clearly more work in this important direction is needed.

Finally, a similar mass decomposition holds for the pion at the same soft renormalization scale of $\bar{\rho}=0.6 \mathrm{GeV}$, with the estimates,

$$
\begin{aligned}
& \frac{M_{Q}^{\pi}}{m_{\pi}} \approx \frac{3}{8} \frac{1}{1+\kappa} \approx 34 \% \\
& \frac{M_{G}^{\pi}}{m_{\pi}} \approx \frac{3}{8} \frac{\kappa}{1+\kappa} \approx 3 \% \\
& \frac{M_{A}^{\pi}}{m_{\pi}}=\frac{1}{8} \approx 13 \% \\
& \frac{M_{m}^{\pi}}{m_{\pi}}=\frac{1}{2} \approx 50 \% .
\end{aligned}
$$

About $85 \%$ of the pion mass stems from the valence quarks (hopping zero modes), $13 \%$ from the gluon condensate or epoxy (displaced vacuum instanton field), and 3\% from emerging valence gluons. Needless to say that all mass contributions in the pion vanish smoothly in the chiral limit. Again, quantum evolution will enhance $M_{G}^{\pi}$ at the expense of $M_{Q}^{\pi}$, with effectively dressing $\kappa \approx 0.1 \rightarrow 0.5$ at $\mu=2 \mathrm{GeV}$.

\section{MEASURING THE QCD VACUUM COMPRESSIBILITY}

While the present discussion has focused on some key aspects of the QCD vacuum and the hadronic mass sum rule, it is worth noting that the results (12)-(18) can be recast in the following form:

$$
\frac{\left\langle P\left|F^{2}(0)\right| P\right\rangle}{\left(4 \pi\left(m_{N}-\sigma_{\pi N} / 2\right)\right)^{2}} \approx-\sigma_{F^{2}},
$$

with the QCD vacuum compressibility,

$$
\sigma_{F^{2}}=\frac{1}{32 \pi^{2}} \int d^{4} x \frac{\left\langle F^{2}(x) F^{2}(0)\right\rangle_{C}}{\left\langle F^{2}(0)\right\rangle} .
$$

A measure of the gluon condensate or epoxy inside the proton (left hand-side) is a measure of the QCD vacuum compressibility $\sigma_{F^{2}}$ (right hand-side), modulo 
the pion-nucleon sigma term, which is small. Since (32) is a nucleon connected matrix element, it is natural that it probes the fluctuations of $F^{2}$. While in the vacuum state, the gluon condensate is positive, (33) shows that it is negative in the nucleon state. The nucleon state carries less epoxy.

The cooled Yang-Mills vacuum in Fig. 1 is composed of interacting topological charges. The vacuum compressibility $\sigma_{F^{2}}$ captures the squared variance of their interactions: $\sigma_{F^{2}}=1$ for a noninteracting gas phase, $\sigma_{F^{2}}<1$ for an interacting liquid phase, and $\sigma_{F^{2}} \ll 1$ for a strongly interacting crystal phase. QCD low-energy theorems suggest $\sigma_{F^{2}} \approx 4 / b \approx 4 / 11$ (one loop and quenched) [15], so the QCD instanton vacuum appears to be a dilute quantum topological liquid. A measure of $\sigma_{F^{2}}$ is a measure of a fundamental and universal parameter of the QCD vacuum.

\section{CONCLUSIONS}

The QCD instanton vacuum is populated with topological tunneling configurations, each costing zero energy. The way a light quark can propagate coherently through this maze of tunneling configurations is through its zero mode, scattering and hopping from an instanton to an antiinstanton and so on. The scattering through the instanton flips chirality, an amazing effect caused by a nonperturbative vector interaction (a perturbative gluon interaction preserves chirality). The hopping generates a very dense band in the virtual quark spectrum, reminiscent of the conduction band in conductors. As a result, chiral symmetry is spontaneously broken, a chiral condensate is formed, and a running constituent quark mass emerges.

The QCD instanton vacuum breaks simultaneously conformal symmetry, with a large and negative vacuum energy density or equivalently, a large and positive gluon condensate (gluon epoxy). A hadronic excitation in this vacuum, whether a quark, a meson, or a baryon costs energy or mass. A useful and physical way to budget this mass is Ji's mass decomposition of the energy momentum tensor $[9,22]$. In the QCD instanton vacuum, we find that the hadronic masses are largely due to the contribution of the valence quarks as they hop and drag the gluon epoxy.

Finally, a measure of the gluon condensate or epoxy in the nucleon is a measure of the compressibility of the QCD instanton vacuum as a topological liquid. The diluteness of this liquid is central in our nonperturbative understanding of the emergence of mass in QCD using analytical methods. This gluonic content of the proton may be accessible through threshold electromagnetic production of heavy quarkonia [17] and perhaps diffractive cluster production in hadron-hadron collisions [31] at current and future collider facilities.

\section{ACKNOWLEDGMENTS}

I thank Xiang-dong Ji, Zein-Eddine Meziani and Edward Shuryak for discussion. This work is supported by the Office of Science, U.S. Department of Energy under Contract No. DE-FG-88ER40388.
[1] D. Diakonov, Chiral symmetry breaking by instantons, Proc. Int. Sch. Phys. Fermi 130, 397 (1996).

[2] T. Schfer and E. V. Shuryak, Instantons in QCD, Rev. Mod. Phys. 70, 323 (1998).

[3] M. A. Nowak, M. Rho, and I. Zahed, Chiral Nuclear Dynamics (World Scientific, 1996).

[4] J. Greensite, Confinement from center vortices: A review of old and new results, EPJ Web Conf. 137, 01009 (2017).

[5] E. V. Shuryak, Probing the boundary of the nonperturbative QCD by small size instantons, arXiv:hep-ph/9909458.

[6] M. C. Chu, J. M. Grandy, S. Huang, and J. W. Negele, Correlation functions of hadron currents in the QCD vacuum calculated in lattice QCD, Phys. Rev. D 48, 3340 (1993).

[7] J. J. M. Verbaarschot and I. Zahed, Spectral Density of the QCD Dirac Operator Near Zero Virtuality, Phys. Rev. Lett. 70, 3852 (1993).

[8] H. Wittig, QCD on the lattice, in Particle Physics Reference Library: Volume 1: Theory and Experiments, edited by H. Schopper (Springer, 2020), pp. 137-262.
[9] X.-D. Ji, A QCD Analysis of the Mass Structure of the Nucleon, Phys. Rev. Lett. 74, 1071 (1995).

[10] D. B. Leinweber, Visualizations of the QCD vacuum, in Workshop on Light-Cone QCD and Nonperturbative Hadron Physics, edited by A. W. Schreiber and A. G. Williams (World Scientific, 1999), pp. 138-143.

[11] J. C. Biddle, W. Kamleh, and D. B. Leinweber, Visualization of center vortex structure, Phys. Rev. D 102, 034504 (2020).

[12] E. V. Shuryak, The role of instantons in quantum chromodynamics. 1. Physical vacuum, Nucl. Phys. B203, 93 (1982).

[13] D. Diakonov, M. V. Polyakov, and C. Weiss, Hadronic matrix elements of gluon operators in the instanton vacuum, Nucl. Phys. B461, 539 (1996).

[14] M. Kacir, M. Prakash, and I. Zahed, Hadrons and QCD instantons: A Bosonized view, Acta Phys. Pol. B 30, 287 (1999).

[15] V. A. Novikov, M. A. Shifman, A. I. Vainshtein, and V. I. Zakharov, Are All Hadrons Alike? DESY-check = Moscow Inst. Theor. Exp. Phys. Gkae-Itef-81-048 (81,rec.jun.) 32P 
and Nucl. Phys. B191 (1981) 301-369 and Moscow Inst. Theor. Exp. Phys. Gkae-Itef-81-042 (81,rec.apr.) $70 P$. (104907), Nucl. Phys. B191, 301 (1981).

[16] D. E. Kharzeev, The mass radius of the proton, arXiv:2102.00110 [Phys. Rev. D (to be published)].

[17] S. Joosten and Z.E. Meziani, Heavy quarkonium production at threshold: From JLab to EIC, Proc. Sci., QCDEV2017 (2018) 017.

[18] K. A. Mamo and I. Zahed, Diffractive photoproduction of $J / \psi$ and $\Upsilon$ using holographic QCD: gravitational form factors and GPD of gluons in the proton, Phys. Rev. D 101, 086003 (2020).

[19] J. V. Steele, H. Yamagishi, and I. Zahed, The Pion-nucleon sigma term and the Goldberger-Treiman discrepancy, arXiv: hep-ph/9512233.

[20] C. Alexandrou et al., Nucleon axial and pseudoscalar form factors from lattice QCD at the physical point, Phys. Rev. D 103, 034509 (2021).

[21] M. Hoferichter, J.R. de Elvira, B. Kubis, and U.-G. Meißner, Remarks on the pion-nucleon $\sigma$-term, Phys. Lett. B 760, 74 (2016).

[22] X.-D. Ji, Breakup of hadron masses and energy-momentum tensor of QCD, Phys. Rev. D 52, 271 (1995).
[23] C. Lorcé, On the hadron mass decomposition, Eur. Phys. J. C 78, 120 (2018).

[24] C. D. Roberts, On mass and matter, arXiv:2101.08340.

[25] A. Metz, B. Pasquini, and S. Rodini, Revisiting the proton mass decomposition, Phys. Rev. D 102, 114042 (2020).

[26] X. Ji, Proton mass decomposition: Naturalness and interpretation, Front. Phys. 16, 64601 (2021).

[27] Y.-B. Yang, J. Liang, Y.-J. Bi, Y. Chen, T. Draper, K.-F. Liu, and Z. Liu, Proton Mass Decomposition from the QCD Energy Momentum Tensor, Phys. Rev. Lett. 121, 212001 (2018).

[28] X. Ji and Y. Liu, Quantum anomalous energy effects on the nucleon mass, Sci. China Phys. Mech. Astron. 64, 281012 (2021).

[29] A. Athenodorou, Ph. Boucaud, F. De Soto, J. RodríguezQuintero, and S. Zafeiropoulos, Instanton liquid properties from lattice QCD, J. High Energy Phys. 02 (2018) 140.

[30] M. Luscher and P. Weisz, Perturbative analysis of the gradient flow in non-abelian gauge theories, J. High Energy Phys. 02 (2011) 051.

[31] E. Shuryak and I. Zahed, How to observe the QCD instanton/sphaleron processes at hadron colliders?, arXiv: 2102.00256 . 